\title{
SOFT X-RAY MEASUREMENTS ON THE PLT TOKAMAK
}

S. VON GOELER, N, SAUTHOFF, M. BITTER, K. BRAU, D. EAMES,

B. FRAENKEL, A, GREENBERGER, K. HILL, R. HORTON, G. HOVEY, J. HOVEY, W. RONEY, AND $W$, STODIEK

\section{PLASMA PHYSICS LABORATORY}

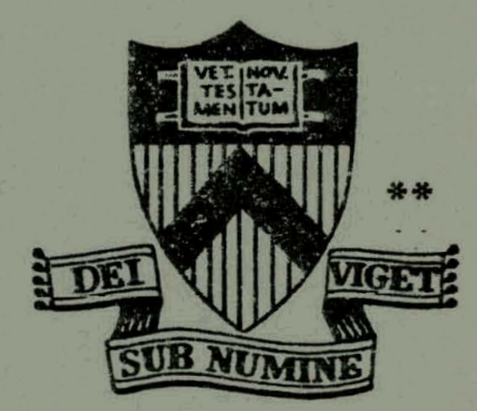

\section{PRINCETON \\ UNIVERSITY PRINCETON, NEW JERSEY}

This work was supported by U. S. Energy Research and Development Administration Contract EY-76-C-02-3073. Reproduction, translation, publication, use and disposal, in whole or in part, by or for the United States Government is permitted. 


\section{DISCLAIMER}

This report was prepared as an account of work sponsored by an agency of the United States Government. Neither the United States Government nor any agency Thereof, nor any of their employees, makes any warranty, express or implied, or assumes any legal liability or responsibility for the accuracy, completeness, or usefulness of any information, apparatus, product, or process disclosed, or represents that its use would not infringe privately owned rights. Reference herein to any specific commercial product, process, or service by trade name, trademark, manufacturer, or otherwise does not necessarily constitute or imply its endorsement, recommendation, or favoring by the United States Government or any agency thereof. The views and opinions of authors expressed herein do not necessarily state or reflect those of the United States Government or any agency thereof. 


\section{DISCLAIMER}

Portions of this document may be illegible in electronic image products. Images are produced from the best available original document. 
NOTICE

This report was prepared as an account of work sponsored by the United States Government. Neither the United States nor the United States Energy Research and Development Administration, nor any of their employees, nor any of their contractors, subenntractors, or their employees, makes any warranty, express or implied, or assumes any legal liability or responsibility for the accuracy, completeness or usefulness of any information, apparatus, product or process disclosed, or represents that its use would not infringe privately owned rights.

Printed in the United States of America.

Available from

National Technical Information Service

U. S. Department of Commerce 5285 Port Royal Road

Springfield, Virginia 22151

Price: Frinted Copy $\$ \ldots$; Microfiche $\$ 3.00$

*Pages

$1-50$

$51-150$

$151-325$

$326-500$

501-1000
NTIS

Selling Price

$\$ 4.00$

5.45

7.60

10.60

13.60 
S. von Goeler; N. Sauthoff, M. Bitter, K. Brau, D. Eames, B. Fraenkel*, A. Greenberger, K. Hill**, R. Horton, G. Hovey, J. Hovey, W. Roney, and W. Stodiek

$$
\begin{gathered}
\text { Plasma Physics Laboratory, Princeton University } \\
\text { Princeton, New Jersey 08540, USA }
\end{gathered}
$$

PPPI -1383

October 1977

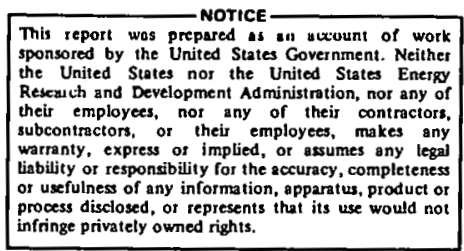

This repart was prepared as an wluount of work the United States nor the United States Energy subcontractors, their warranty, express of implied, or assumes any legal at its use would not

$+$

Presented at the 2nd Conference on Hot Plasma Diagnostics, Kharkov, USSR, 22-27 September 1977 . 
S. von Goeler, N. Sauthoff, M. Bitter. K. Brau D. Eames, B. Fraenkel*, A. Greenberger, K. Hill**,

R. Horton, G. Hovey, J. Hovey, W. Roney and W. Stodiek

Plasma Physics Laboratory, Princeton University Princeton, New Jersey 08540, USA

As the temperature in toroidal confinement devices increases, an enlarging fraction of the electromagnetic radiation is emitted in the $x$-ray region. The $x$-ray emission therefore becomes an increasingly important feature from the standpoint of energy loss as well as from the diagnostic viewpoint. In this paper four experiments are described that.currently run on the PLT tokamak and which utlize the soft $x$-ray emission of the plasma as a diagnostic: the pulse height analysis system for temperature and impurity measurements; the curved crystal Bragg spectrometer for the determination of ionization states of impurities; "windowless" surface barrier detectors for the investigation of the ultra soft $x$-ray radiation in the energy range $0.1 \mathrm{keV}<\mathrm{hv}<1 \mathrm{keV}$ and a silicon diode array for $\mathrm{x}$-ray fluctuation measurements. For each diagnostic we give a short technical description, and then discuss some recent results obtained with it on PLT in order to demonstrate its use.

As we shall proceed with the detailed description of the experiments, one may notice by several indicators that the $x$-ray diagnostics of toroidal plasmas have reached a state of maturity. For the older, established techniques, one finds a high degree of technical sophistication: multidetector systems and fancy computer data acquisition are employed. There is a striving for the highest accuracy, as in the crystal spectrometer measurements, and a push toward the last frontiers, such as the technical difficult, "very soft $x$-ray region.

\section{The Pulse Height Analysis System}

The pulse height analysis (PHA) of $x$-rays belongs to the old, established techniques [1]. It gives an overview of the $x$-ray emission in the energy range 1 to $20 \mathrm{keV}$, and provides a measurement of the plasma electron temperature and the impurity concentration of oxygen, chromium, iron, and nickel.

\footnotetext{
* The Racah Institute of Physics, The Hebrew University, Jerusalem, Israel. お丈

On leave from Oak Ridge National Laboratory, Oak Ridge, TN 37830 .
} 
As detectors, one uses liquid nitrogen-cooled, lithium-drifted silicon detectors. A single photon creates electron-hole pairs in the silicon, i.e., a charge pulse, which is proportional to the photon energy. The pulses are sorted out according to energy, and counted in the pulse height analyzer. There is beautiful equipment available for this technique from the nuclear instrumentation industry. The main technical difficulty is encountered with the pulse pile-up problem [2]. Because of the finite duration of a discharge and the requirement to obtain good statistics, one would like to operate at the highest possible countrates. The countrate is limited because simultaneous arrival of two photons at the detector creates "false" double pulses, which distort the spectrum in the high-energy region where there are only few good pulses due to the sharp, Maxwellian fall-off of the photon spectrum. The difficulties with pulse pile-up can be alleviated with a multi-detector system (Fig. 1). The high-energy region, where pulse pile-up occurs, is measured by a different detector which has a thick foil and large aperture in front of it. Changes in plasma electron temperature cause considerable variations in the countrate of the high-energy detector. To accomodate these variations, we use three high-energy detectors on PLT.

A schematic of the system as installed on PLT is shown in Fig. 2. For each discharge, data are taken during 14 time intervals, which makes a total of 56 spectra. After a shot, the data are processed by the PDP-10 computer: first, the spectra from the four detectors are synthesized into one spectrum, corrected for foil attenuation, detector efficiency, and geometry (Fig. 3). Then a polynomial in $E$ is fitted to the logarithm of the $x$-ray continuum, and its coefficients are stored in the computer for further evaluation; counts under

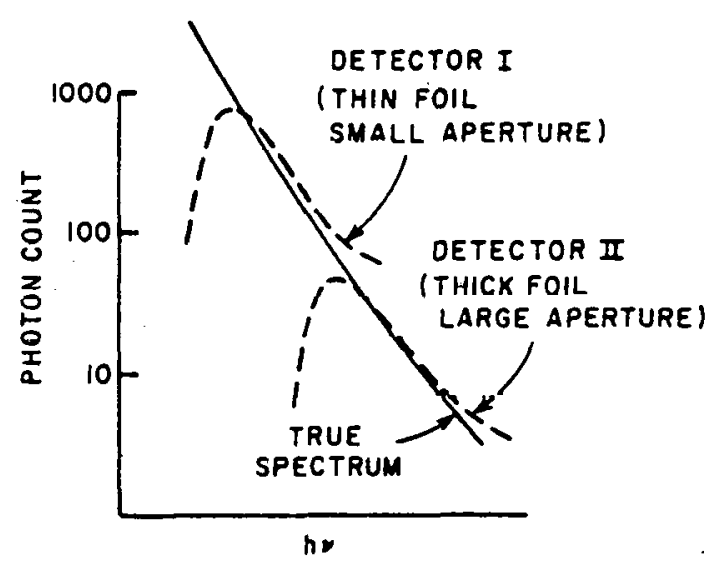

Fig. 1. Schematic ilzustrating how multidetector. FHA=systems avoid problems associated with pulse pile-up: the energy region, where pile-up occurs in detector $I$ is measured with detector II. (PPPL-773848) prominent impurity lines, like $k \alpha$, are also stored. Then the apparatus is moved between shots for a radial scan. The next step is to Abel invert the continuum, using the polynomials, and to invert the impurity line intensities. We now have local $x$-ray spectra for evaluation, and can determine a) the electron temperature from the slope of the continuum (Fig. 4ai, 


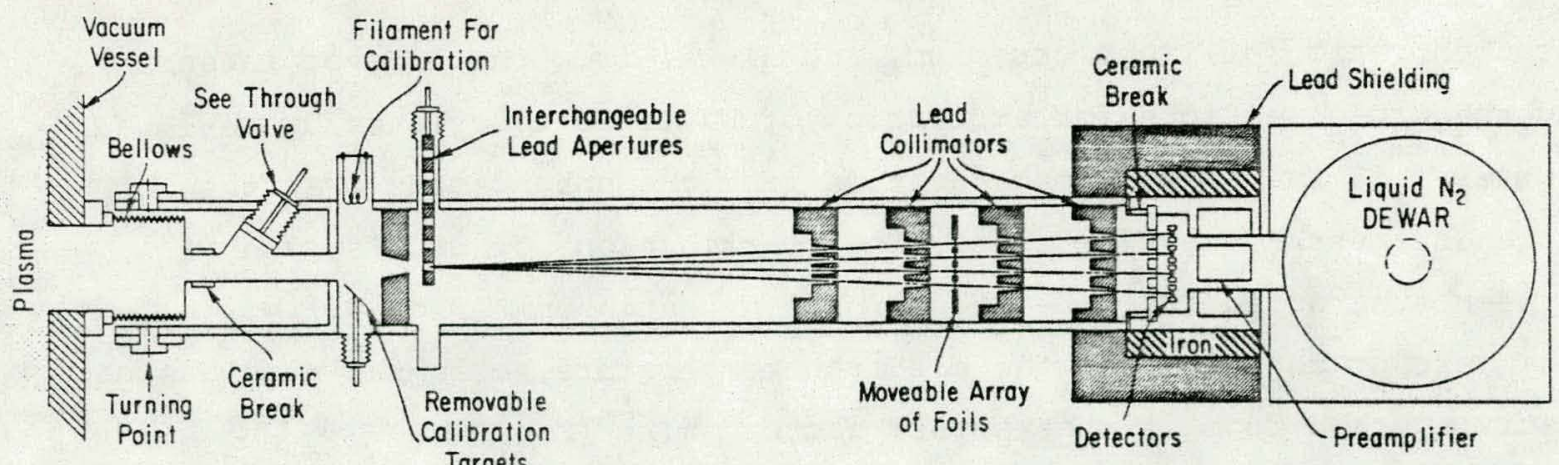

(Al, Ti, Cu)
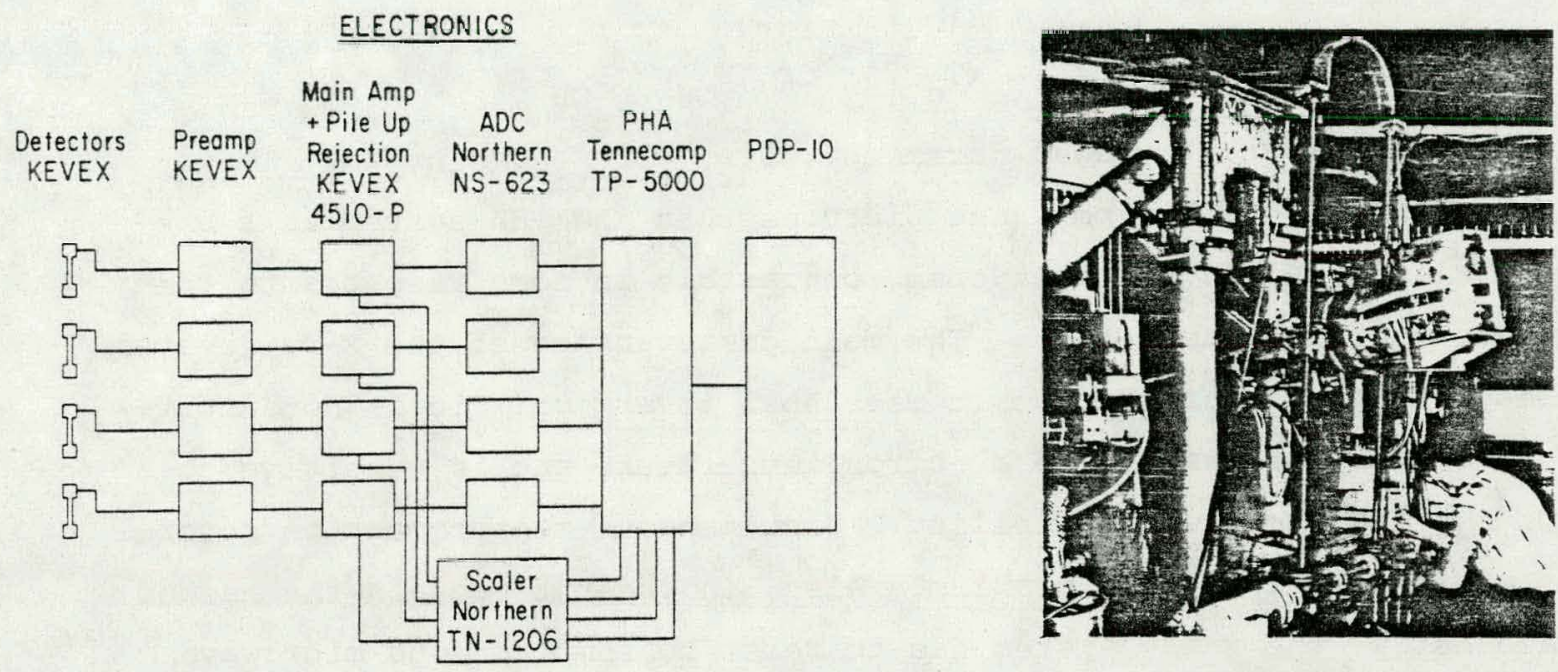

Fig. 2. Schematic of the multidetector-PHA-system installed on PLT. (PPPL-773872)
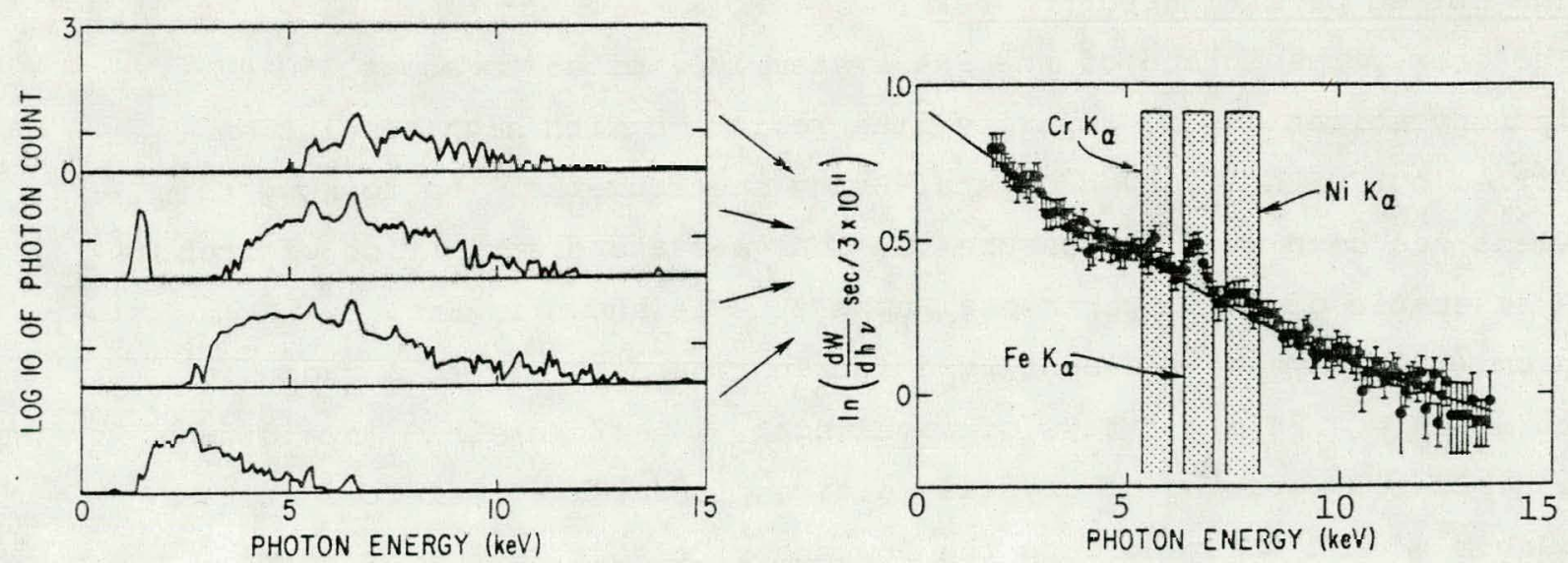

Fig. 3. Row data from four detectors (a) are synthesized into one $x$-ray spectrum $(b)$. The smooth curve represents the polynomial fit tc the continum. The energy ranges of the chrome, iron, and nickel $K a$ lines are indicated. (PPPL-773858) 
the concentration of iron, chromium, and nickel from the line of intensity, using the cross sections for excitation of $\mathrm{K} \alpha$ lines calculated by Merts [3] (Fig. 4e), c) the oxygen concentration from the enhancement over the hydrogen bremsstrahlung [1] (after the contributions of $\mathrm{Cr}, \mathrm{Fe}$, and $\mathrm{Ni}$ have been subtracted from the recombination continuum) (Fig. 4f),

d) the current density from the electron temperature, assuming neoclassical resistivity, and from it, the safety factor $q(r)$ (Fig. $4 b$ ), e) the uncorrected electron-energy-confinement time from the electron temperature,

$$
\tau_{E}^{\prime}=\int(3 / 2) n_{e} k T_{e} 2 \pi r d r / V_{O H} \cdot I_{O H}
$$

(Fig. 4C), and (f) the runaway parameter, $v_{\text {drift }} / V_{\text {crit }}$ (Fig. 4d).

It becomes evident from these pictures that the PHA system is a useful general plasma diagnostic system, comparable in some respects to the Thumson-scattering measurement. The main disadvantage of the $x$-ray system compared with the laser is that it uses Abel inversion: it is not accurate for hollow profiles of the electron temperature and is sensitive to lack of shot to shot reproducibility. Simultaneous measurement on several chords would alleviate this problem. Also, in order to obtain the needed plasma density, the $x$-ray system has to refer to simultaneous microwave density measurements. On the other hand, the PHA system provides temporal evolution of the temperature at a fixed radius, a wealth of information on impurities, and indicates the presence of electron runaway tails.

\section{The Curved Crystal Spectrometer}

The energy resolution of the PHA system is limited to about $150 \mathrm{eV}$. Detailed investigations of impurity line radiation with high resolution are carried out with a curved crystal-Bragg spectrometer. Up to now, this instrument has been used to measure the charge-state distribution of iron.

A schematic of the experimental apparatus is shown in Fig. 5. A germanium (220) crystal was bent by a four-bar bending jig to a radius of curvature $2 k=4.04 \mathrm{~m}$. X rays of wavelength $\lambda=(2 d / n) \sin \theta \quad(n=d i f-$ fraction order, $d$ =spacing of crystal lattice planes) are reflected at the Bragg angle $\theta$ and focussed onto the Rowland circle of radius $R$. The photons are detected by a position-sensitive, multiwire proportional counter (Fig. 6) [4]. A photon produces an avalanche to an anode wire. The image charges are picked-up by the cathode wires and transmitted to a delay line. Standard electronics convert the difference in arrival time of the charge 

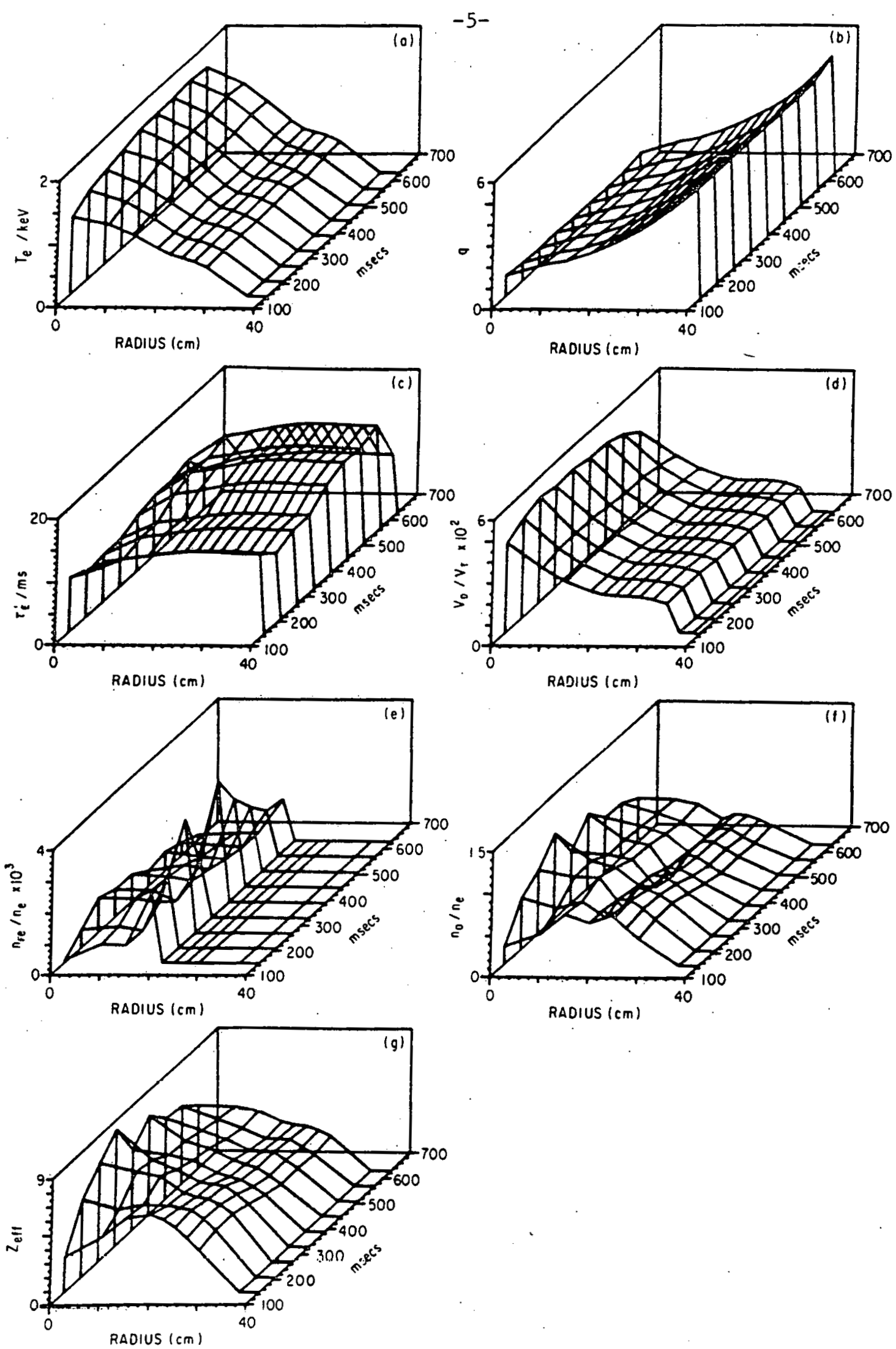

Fig. 4. Results from the PHA-System after a radial scan. The dataare typical for a hydrogen discharge with a plasma current $I_{o m}=375 \mathrm{kA}$,
a toroidal field $B T=32 \mathrm{kG}$, and a central density $n_{0}=3.5 \times 10^{13} \mathrm{~cm}^{-3}$. Simizar data for a high density he Lium dicoharge were presented at the Berchtesgaden conference. (PPPL-773866) 
pulse at the two ends of the delay line into a pulse, the amplitude of which depends linearly on the position at which the photon struck the detector. The pulses are. fed into a pulse-height analyzer and from there into the PDP10 computer for evaluation. Resolution of the instrument, determined from the width of the iron $K \alpha$ line at $6.4 \mathrm{keV}$, was approximately $3.4 \mathrm{eV}$ FWHM.

Implurity ions iri tukamaks axe located in shells
CURVED - CRYSTAL SPECTROMETER

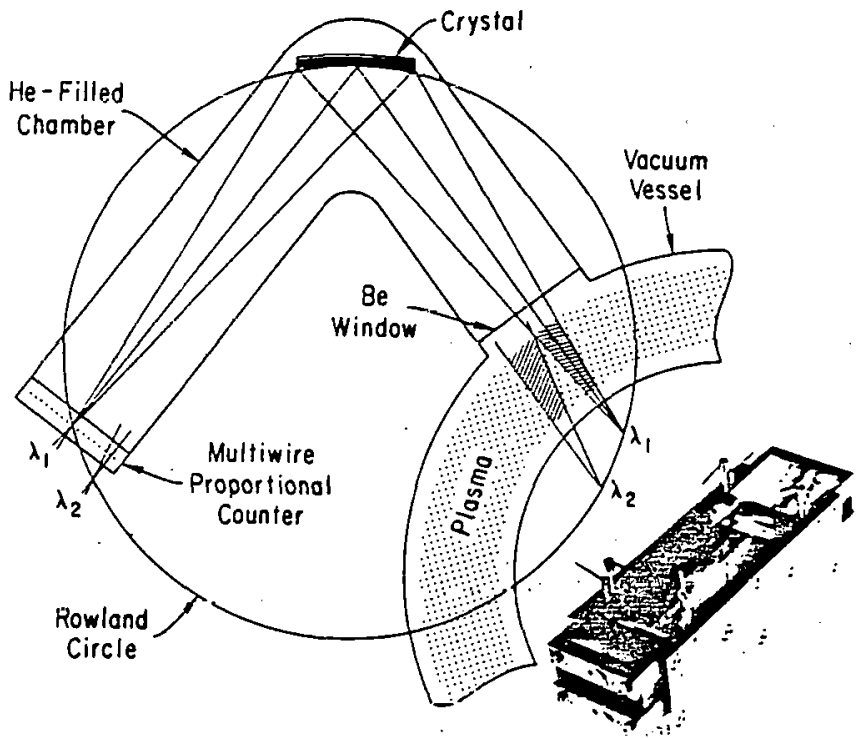

Fig. 5. Sohemativ of the Bragg owneucrystal spectrometer. The photo shows the jig for bending the crystal. (PPPL-773846) of different charge state around the center uf the discharge. It is necessary to know the charge state distribution in order to acuurately determine the concentration and radial variation of an impurity by line radiation. Experimentally, the charge state distribution of iron can be determined from the energy shift of the $1 \mathrm{~s}-2 \mathrm{p}$ transition relative to the $\mathrm{k} \alpha \mathrm{line}$ of unstripned iron [5]. A major reason for using this transition is that very detailed ab initio calculations have been performed for it by. Mert.s [3], allowing us to make quantitative comparisons between theory and experiment.

A comparison between a measured and a calculated spectrum is shown in

Fig. 7. The theoretical

spectrum was shifted toward

MULTIEIRE POOPOATIONLL COUMTE ANO ELECTRONICS

lower energy by only $11 \mathrm{eV}$ $(<0.28)$, indicating the remarkable accuracy of the calculations. There is dimost a vile-to-one correspondence, not only for the major peaks, corresponding to different charge stales indicated by Roman numerals, but also for the subpeaks. In Fig. 8, we show a series

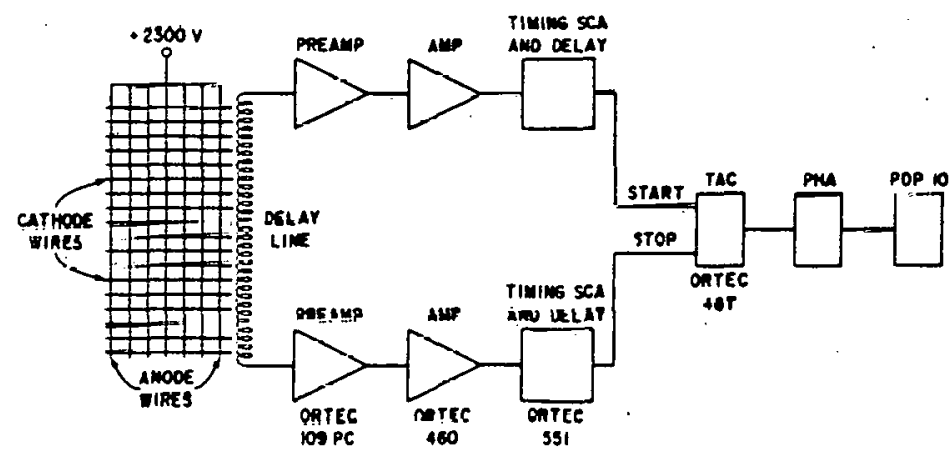

Fig. 6. Schematic illustrating the operation of the multiwire proportional counter. The instrument used on PLT was built by Gunman Aerospace Corporation [4]. (PPPL-773603) 


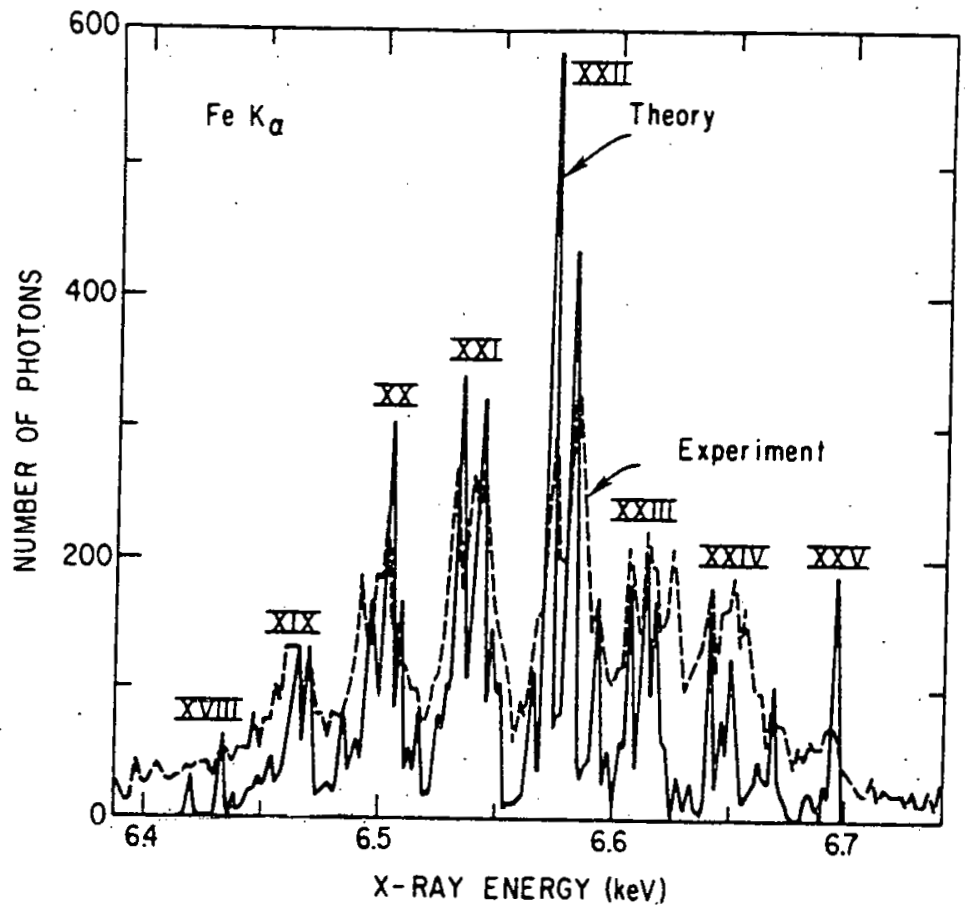

Fig. 7. Comparison of a typical Fe-Ka spectmon from PLT with a theoretical spectrum generated by Merts [3]. (PPPL-773714) of measured spectra of the iron $1 s-2 p$ transition, arranged according to electron temperature as measured with the PHA system. The predominant charge state increases from FeXXI to FeXXV as the temperature increases from 800 to $1800 \mathrm{eV}$.

Actually, it is not possible to associate a major peak exactly with a given charge state. Multiplet. splitting results in a structure within each major peak. More importantly, an ion in a given charge state, e.g.,

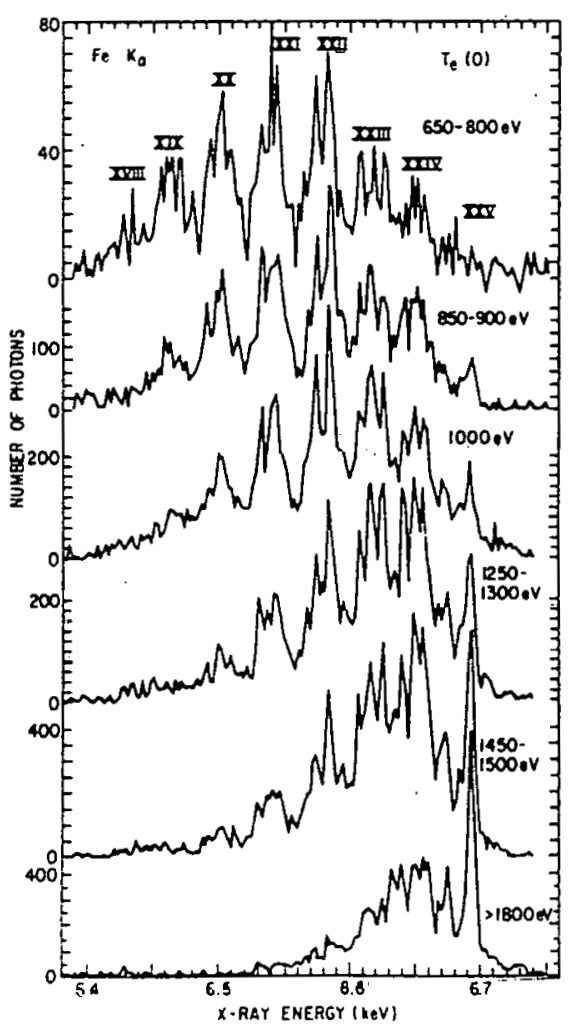

Fig. 8. Scomple Fe Ka spectra at different temperatures izlustrate the shift to higher ionization states as teimperature inceases. (PPPL-773660)
FeXXII, can produce radiation in the neighboring FeXXI and FeXXIII peaks. The main transition from collisional excitation is $1 s^{2} 2 s^{2} 2 \mathrm{p}$ $+e \rightarrow 1 s 2 s^{2} 2 p+1 s^{2} 2 s^{2} 2 p$. However, another possibility is $1 s^{2} 2 s^{2} 2 p \rightarrow 1 s 2 s^{2} 2 p n l \rightarrow 1 s^{2} 2 s^{2} n l$ with $n \geq 3$. Since the energy shift of the $(1 s-2 p)$ transition depends mainly on the number of I-shell electrons, this latter transition falls under the FeXXIII peak. Similarly, dielectronic excitation, which actually dominates over collisional excitation at present tokamak temperatures, can produce radiation from FeXII with final ground states $1 s^{2} 2 s^{2} 2 p^{2}$, $1 s^{2} 2 s^{2} 2 p n l$, and $1 s^{2} 2 s^{2} n l n ' l^{\prime}$ (with $n, n ! \geq 3$ ), which fall-under the basic peaks of FCXXIII, FeXXII; and FeXXIII. In order to determine the major peaks of the spectra, we have to perform a matrix inversion. Unfortunately, the computer program executing the matrix inversion is not yet operational. As a preliminary evaluation, we have comparea the 
intensities under a charge state peak with theoretical predictions [3]. In Fig. 9 the fractional intensities under a peak are plotted vs. temperature. In the top graph the experimental data are presented; in the bottom graphs, Cowan's theoretical calculations based on corona equilibrium using ionization crossections of Lotz [6] and Griem [7] (one-half the Lotz value for L-electrons), respectively. Limited space does not allow us to give a detailed discussion of the differences between theory and experiment. At $800 \mathrm{ev}$, the experiment agrees with calculations based on the Lotz ionization cross sections whereas at $1600 \mathrm{eV}$ there is better agreement with the Griem values. Within these uncertainties, the iron in the center of the discharge is in corona equilibrium.

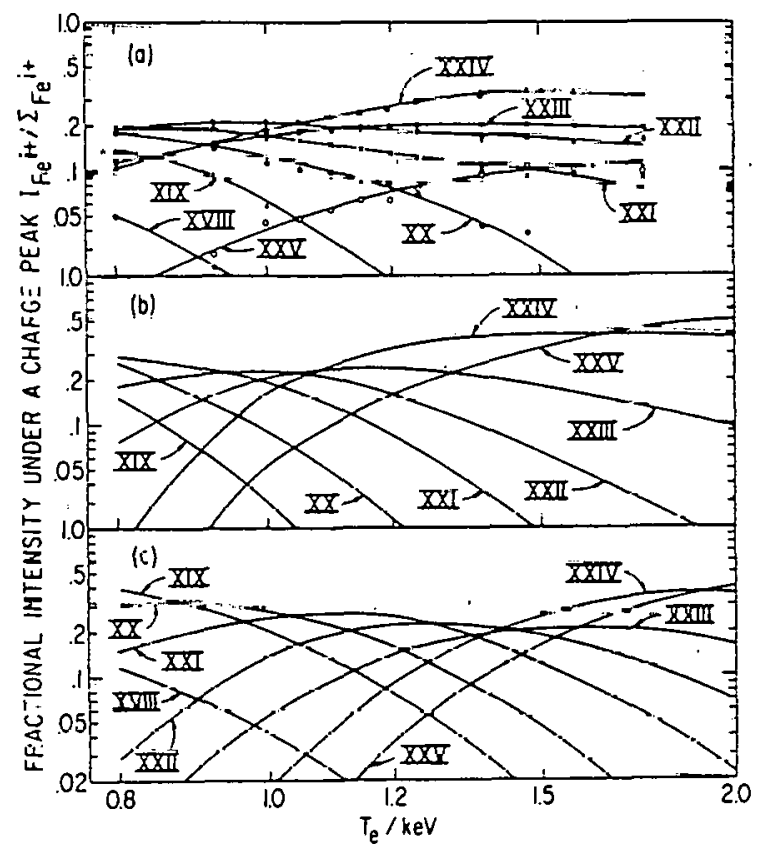

Fig. 9. Fractional intensity under a charge state peak vs. electron temperature. (a) experimental values, (b) and (c) theoretical predictions [3], using different ionization cross sections in the calculation of the corona equilibrium. (PPPL-773859)

III. The Ultra-Soft $\mathrm{x}$-Ray Region $(0.1 \mathrm{keV}<\mathrm{h} v<1 \mathrm{keV})$

Tia main radiation power loss in tokanaks is emitted in the ulera-coft $x$-ray $(i j x)$ region. The recent observations of hollow radial profiles of the electron temperature [8]. suggest that in the center of the discharge the radiative power loss is comparable to or larger than the ohmic heating power input, and has stimulated intense investigation of this region. These investigations were carried out concurrently by the spectroscopists using a grazing-incidence spectrometer and with x-ray techniques using foil absorber techniques. 
It was the aim of the ultra-soft $x$-ray experiment to measure an overall broad-band power loss in the energy range $0.1 \mathrm{keV}<\mathrm{hv}<1 \mathrm{keV}$. We used silicon surface-barrier diodes as detectors. In these detectors an incoming photon creates electron-hole pairs (efficiency: I pair per $3.76 \mathrm{eV}$ photon energy). The resulting conduction current in the diode is directly proportional to the energy flux of the radiation. The efficiency of the detector is, however, not quite 100 percent, because of absorption in the gold contact layer $\left(40 \mu \mathrm{g} A \mathrm{Au} / \mathrm{cm}^{2}\right)$ in front of the detector, and possibly in some thin silicon dead layer, located between the gold and the sensitive region. Little is known about the dead layer. Preliminary calibrations suggest a dead layer of $7 \mathrm{\mu g} / \mathrm{cm}^{2} \mathrm{Si}$. The resultant detector absorption is plotted in Fig. 10a. In the same figure, the experimental set-up on PLT is shown. The arrangement includes a set of ultra-thin Be-foils, which enable us to obtain a coarse estimate of the spectral composition of the emitted radiation.

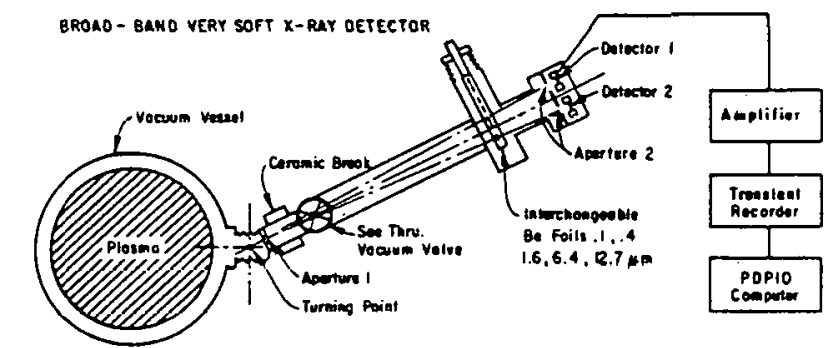

Fig. 10. Schematic drowing of the uitra-soft $x$-ray detector. (a) Transmission coefficient of 0.01 - $10 \mathrm{keV} x$-rays through the detector gold contact layer alone, and through the gold layer plus a thin dead Zayer. (b) Transmission coefficient for the five Be-foils used to obtain coarse energy resolution. (PPPL-7738 $₫ 9$ )

The dominant contribution of the emitted ultra-soft $x$-ray radiation for all measured cases fell into the range $0.15<\mathrm{hu}<0.3 \mathrm{keV}$. The unattenuated USX signal was practically identical to the $50 \AA$ A FU traces as far as the time dependence as well as changes with discharge conditions were concerned. The FUV measurements of Hinnov with higher resolution [9] showed that the radiation consisted of tinree bands of densely spaced 
impurity lines, centered at 33, 50, and $59 \stackrel{\circ}{\mathrm{A}}$, with intensities in the ratio $3: 5: 2$ (Fig. 11). Similar spectra have been seen by Isler [10] on Ormak and in laser plasmas [11] and have been theoretically predicted by cowan [12] to be tungsten $4 d-4 f$ transitions.

Besides the $50 \AA$ A radiation, we also detected higher-energy radiation, namely in the $20 \AA$ and in the $14 \AA$ region with intensities roughly in the ratio $6: 1: 1$ (The intensity and wavelength of the $20 \stackrel{\circ}{\mathrm{A}}$-component is somewhat uncertain due to the intricacies of the foil-absorber method.).

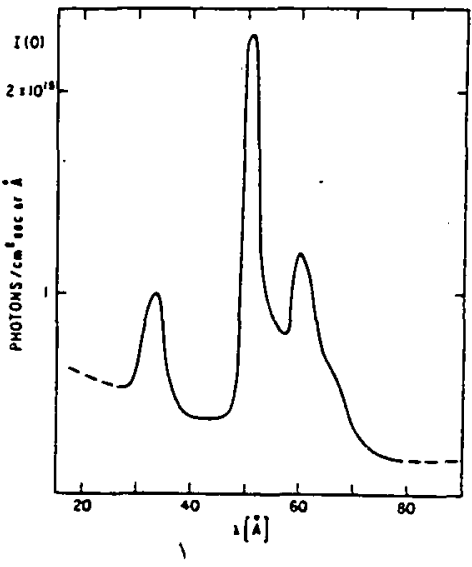

Fig. 11. Typical spectrim of the tungsten radiation measured with the grazing-incidence FUV-spectrometer by $E$. Hinnov. (PPPL-773504)

Typical Abel-inverted radial profiles are shown in Fig. 12. Figures 12a and $b$ show the time-development of the electron temperature and density measured by Thomson scattering of laser light. In this particular discharge,
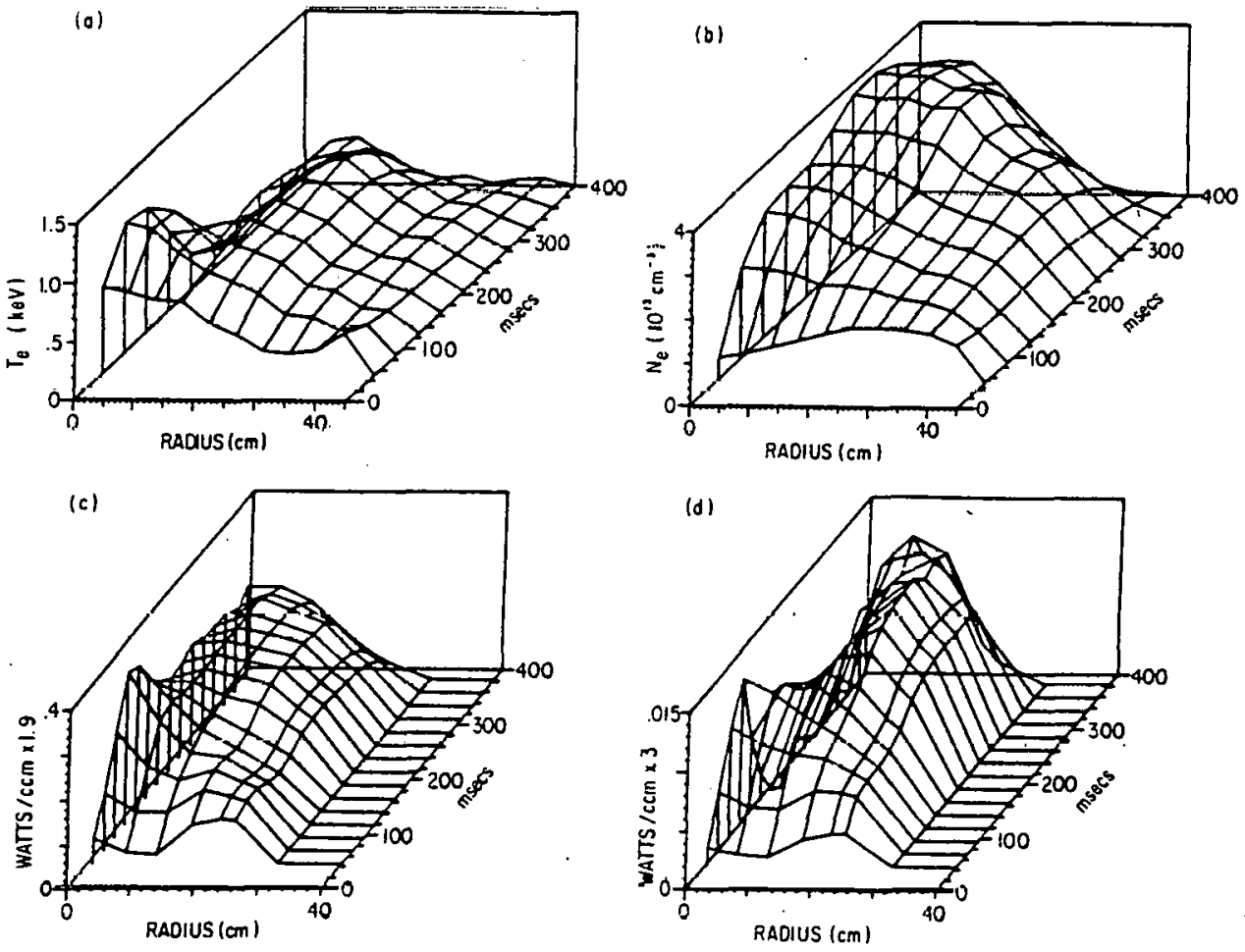

Fig. 12. Time development of a discharge in PLT where tungsten radiation causes the electron temperature profile to turm hollow: (a) and (b) plasma electron temperature and density from Thomson scattering, (c) and (d) Abel inverted USX-signals unattenuated and attenuated by a $6.4 \mathrm{um} \mathrm{Be-}$ foil, respectively. The factors 1.9 and 3.0 on the ordinate label indicate corrections which should be made for detector efficiency. (PPPL-773851) 
the radial temperature profile turned from peaked to hollow and around $150 \mathrm{msec}$; we therefore call it a hollow deuterium discharge. Figures $12 \mathrm{c}$ and $\mathrm{d}$ show the time development of the ultra soft $\mathrm{x}$-ray radiation unattenuated and attenuated by a $6.4 \mu \mathrm{m}$ Be-foil. The low energy cut-off of the foil is $880 \mathrm{eV}(\approx 14 \mathrm{~A})$. The $14 \mathrm{~A}$-component of the radiation, Fig. 12d, follows the laser temperature profile very closely, showing also the transition from peaked to hollow. The tungsten radiation (Fig. $12 \mathrm{C}$ ) exhibits a rather flat profile. The radiated usx-power in the central region $(\approx 400 \mathrm{~mW}$ ) is larger than the ohmic-heating input if we account for the exclusion of the ohmic current due to the low temperature in the center of the discharge. The tungsten radiation seems to provide the energy sink in the center which allows the discharge to remain in a hollow profile. Hollow discharges occurred regularly in PLT at low densities after intensive discharge cleaning. When the discharge was subject to a large oxygen contamination, or when neon was pulsed in, the tungsten radiation was much reduced, and the temperature profile peaked. Another way of obtaining peaked profiles was gas-programming. In the high-density helium discharges ( $\mathrm{n}_{\mathrm{eo}} \approx 1.5 \times 10^{14} \mathrm{~cm}^{-3}$ ) the tungsten radiation level was very small.

\section{X-Ray Fluctuation Measurements}

The transient nature of plasma instabilities and disruptions makes it imperative to measure all quantities simultaneously. On PIT, N. Sauthoff [13] has built an array of silicon surface-barrier detectors, which allows us to measure the time development of radial profiles of the soft $x$-ray emission in one shot. The assembly, sketched in Fig. 13, consists essentially of a slot-hole camera with 20 detectors in the image plane. The photo

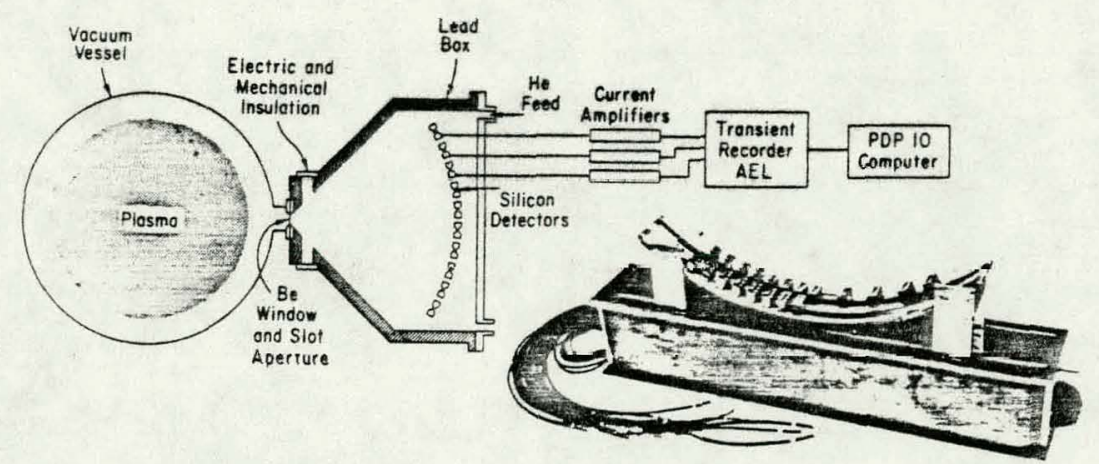

Fig. 13. Schematic drowing of the 20-channel $x$-ray wave detector assembly, showing also a photogroph of the diode assembly. (PPPL-773847) 
current in the detectors is recorded by a 20-channel, 10-bit transient recorder (AEL model PTR-7010) with a maximum digitizing rate of $1 \mathrm{MHz}$, and a $20 \times 4 \mathrm{k}$-word memory. The transient recorder is controlled (e.g., for gain, sampling rate, etc.) by the PDP-10 computer. The timebase is switchable during a plasma shot.

Typical data obtained with the array are presented in Fig. 14. Subfigure a) shows the central trace. The timebase was switched from $2 \mathrm{kHz}$ to $200 \mathrm{kHz}$ during the time interval $189 \mathrm{msec}<\mathrm{t}<199 \mathrm{msec}$, allowing us to investigate in detail a minor disruption occurring at that time. These details are magnified in Fig. 14b. The $\mathrm{x}$-ray intensity vs. time is plotted with the plasma radius as a parameter. We recognize the disruption by the sudden drop in the central x-ray emission at $t=191.2$ msec and the sudden increase of the radiation at larger radii. This feature is interpreted as the sudden onset of an anomalous transport, moving the hot plasma from the center to the outside. Also, we observe precursor oscillations, a larger

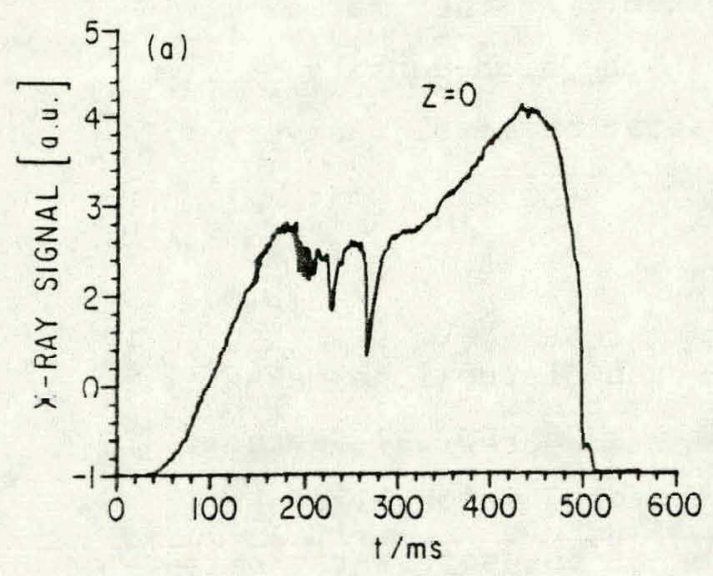

Fig. 14. Data obtained with the wave detector. (a) Response of a single discharge. (b) Response of 14 detectors in the time interval $189.5<t<192 \mathrm{msec}$. The signal from each detector is nomalized so that its maximum and minimum cover the same distance on the ordinate. The slur's on the left indicate the minimum of the signal as well as the plasma position (minimum distance from the line of sight to the plasma center). (c) Response of detectors looking through the plasma $4 \mathrm{~cm}$ above and below the center. (PPPL-773862)
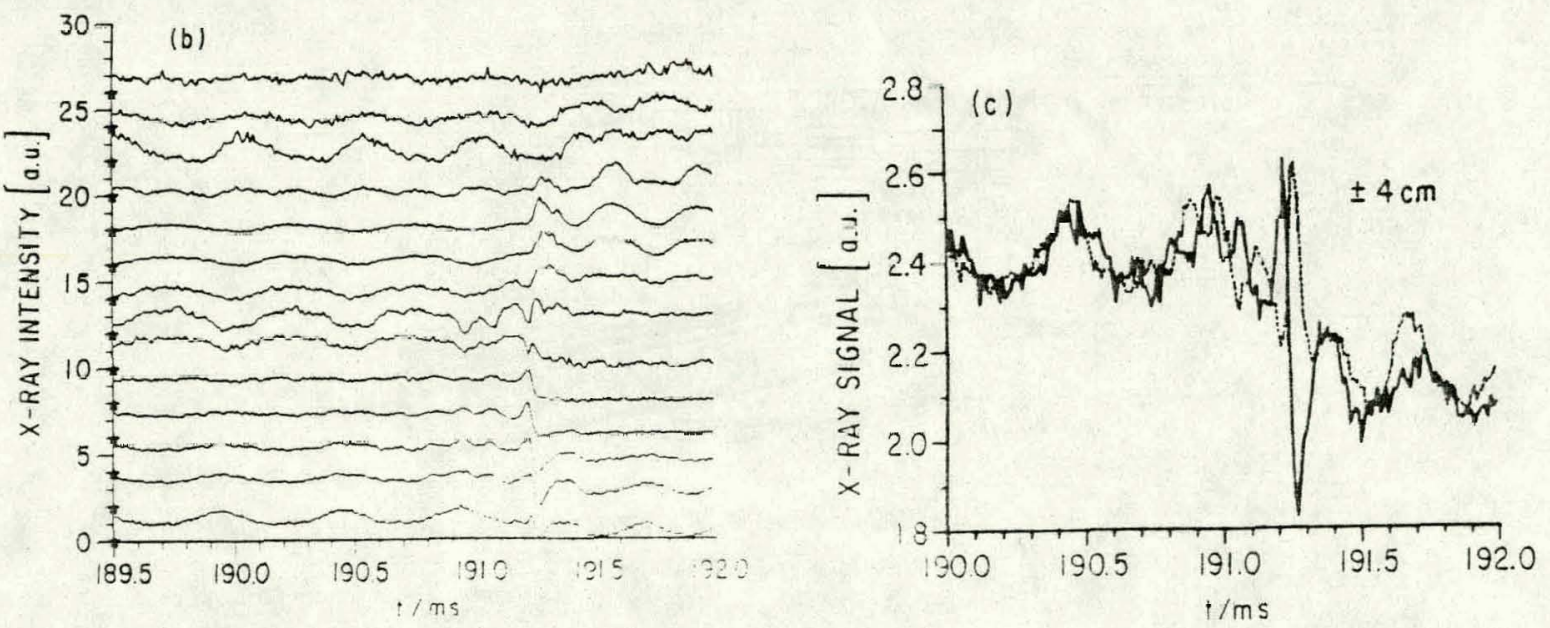
one with a frequency of $2 \mathrm{kHz}$ and a smaller $8 \mathrm{kHz}$ precursor, which comes up around $190.8 \mathrm{msec}$.

unfortunately, the measured signal does not represent a local quantity but corresponds to the $x$-ray emission integrated over a chord of the plasma cross section. It has to be Abel inverted. Because of the helical nature of the fluctuations, the standard Abel inversion for cylindrical columns has to be modified. In Fig. 15, we give the formulae for the first four $m$-modes, where $m$ is the mode number in a Fourier expansion in the poloidal angle, $\phi$. For higher $m$, the special expansion solutions of Ref. 14 have to be used. Actually, we deal here with a special case of image reconstruction techniques [15], encountered, e.g., in brain scans. Figure 15 shows the consequences for the simple case that the local emission of a plasma instability behaves like a step function in radius. Wo see that even modes have an intensity in the center at $r=0$, although the source function vanishes. Furthermore, artificial nodes are created (no node for $m=0$ and 1 , one node for $m=2$ and 3 , two nodes for $m=4$ and 5 , etc.) Actually, these facts are often very helpful in identifying a mode. For example, in the previous Fig. 14b, the large $2 \mathrm{kHz}$ precursor shows up on the center trace and has an artificial node and phase shift at $r=8 \mathrm{~cm}$. It is an $m=2$ oscillation. The $8 \mathrm{kHz}$ higher frequency precursor vanishes on the central trace and has no nodes. It is an $m=1$ mode. This identification is confirmed by Fig. 14c, where we compare signals measured at the two radii $r= \pm 4 \mathrm{~cm}$. As expected from their symetry, the $\mathrm{m}=2$ oscillation is in phase for the two poloidal positions, and the $m=1$ oscillation, which leads directly into the disruption, is out of phase.
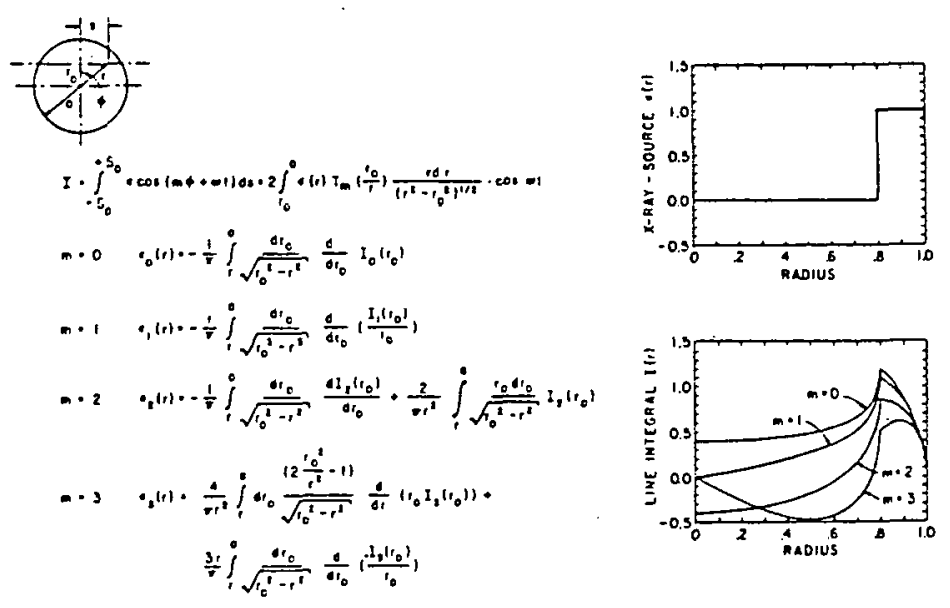

Fig. 15. Formulae for the Abel inversion of line integrated measurements of fluctuations in a colum rotating with frequency w. $I\left(r_{0}\right)$ is the line integrated intensity at radius $r_{0}, \varepsilon_{m}(r)$ is the local source for the component of the fluctuation with poloidal angular dependence $\cos (m \phi+\omega t), T_{m}$ is the Chebyshev polynomial. The graph at lower right shows the response of a line integrating detector to a step source function for poloidal mode numbers $m=0$ to 3 .

(PPPL-773850) 
The procedure for evaluating an oscillation is then to Fourier analyze, in poloidal angle, the chord integral measurements for each radius, Abel invert the different Fourier components, and obtain local emissivity profiles. This has been done in Fig. I6 for an $\mathrm{m}=2$ mode (similar to the $m=2$ precursor of Fig. 14). Lines of constant $x$-ray emissivity are plotted. If the $x$-ray emissivity is constant on a magnetic surface, then we have a plot of magnetic surfaces. The picture marvelously exhibits the island structure of the $m=2$ tearing mode.

It is, unfortunately, not possible to recount all the new results [16] obtained with this technique on PIT. We should, however, mention the work of D. Eames [17], who has constructed an array of USX detectors to study fluctuations of the tungsten radiation. Figure 17 shows one of his first results on disruptions. Comparison is made for a major disruption between the signals from the wave array (Subfig. b) and the ultra-soft array (Subfig. a). During the disruption, the central chords of the wave array decrease, while the outer chords increase. This is traditionally

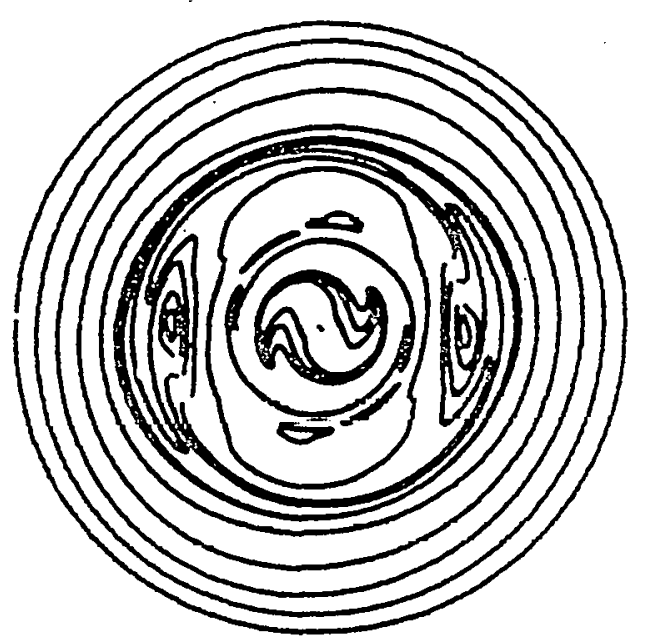

Fig. 16. Abel inversion of an $m=2$ oscillation preceding a dismution measured on PLT with the wave detector. Plottud ure lines of coristuril $x$-ray emissivity $\varepsilon$. (PPPL-773745) interpreted as the sudden onset of some anomolous transport that moves the plasma out to larger radii. By contrast, in the ultra-soft array, all chords increase during the disruption. Since the tungsten radiation is comparable to the ohmic-heating power, the tungsten signal must offset the energy balance of the column. These measurements indicate that the tungsten radiation may play an important role in the development of major disruptions.
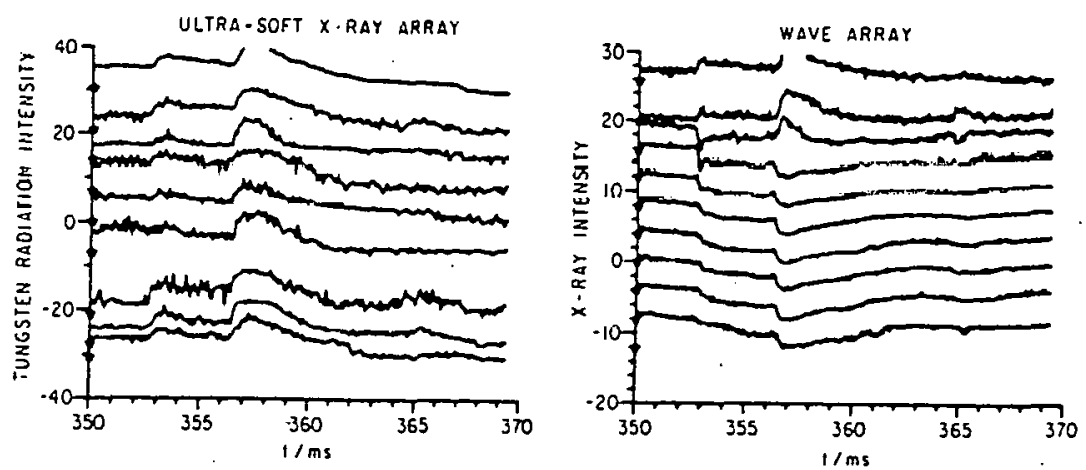

Fig. 17. Measurements of a disruption in PLT. (a) Response of the USXdetector array, (b) response of the wave detector array. The display is the sume as in Fig. 14. (PPPL-773861) 
Acknowledgments: The support of Dr. M. B. Gottlieb and Dr. H. P. Furth is greatfully acknowledged. We thank W. Mycock, M. Ferron and his crew for the technical assistance. We are grateful to Dr. E. Hinnov and Drs. N. Bretz and $D$. Johnson for supplying Fig. 11 and Fig. $12 a$ and $b$, respectively. Dr. R. Cowan has provided us with detailed computer outputs of his calculations. This work was supported by U. S. Energy Research and Development Administration Contract EY-76-C-02-3073.

\section{References:}

[1] S. von Goeler, et al., Nucl. Fusion 15 (1975) 301.

(2) S. von Goeler, et al., Princeton Plasma Physics Laboratory Report MATT-1060 (1974).

[3] A. L. Merts, et al., Los Alamos Scientific Laboratory Report IA-6220-MS (1976).

[4] A. Favale, et al., Grumman Research Department Report RE-423 (1972).

[5] N. Bretz, et al., in Plasma Physics and Controlled Nuclear Fusion Research I (1974) 55 .

[6] W. Lotz, Z. Phys. 206 (1976) 205.

[7] R. U. Datla, et al., Phys. Rev. 14A (1976) 979.

[8] N. Bretz, to be published in Bull. Am. Phys. Soc. 22 .

[9] E. Hinnov, et al., to be published in Proc. Fifth Colloquium on Ultraviolet and X-Ray Spec. Of Astro. and Lab. Plasmas (London, 1977).

[10] R. Isler, et al., to be published in Phys. Lett.

[11] P. G. Burkhalter, et al., Naval Research Laboratory Report 3444 (1977).

[12] R. D. Cowan, Los Alamos Scientific Laboratory Report LA-6679-MS (1977).

[13] N. R. Sauthoff, Proc. Soc. Photo-Optical Inst. Eng. 106 (1977).

[14] A. M. Cormack, J. Appl. Phys. 35 (1964) 2908.

[15] 2. H, Cho [editor], TEEE Trans. Nucl. Sci. NS-21 (1974).

[16] N. R. Sauthoff, et a1., Princeton Plasma Physics Laboratory Report PPPL-1379 (1977).

[17] D. Eames, et al., to be published in Bull. Am. Phys. Soc. 22 (1977). 